\title{
Health data: when children reach the age of consent
}

\author{
Parents give consent for their children's health data to be used in research, but what \\ happens when the children reach adulthood, and how can researchers keep families involved \\ in the meantime?
}

C OVID-19 vaccinations for teenagers have been in the news, which raises questions about parental influence over the decision of children to get vaccinated - or not. In some countries, including the UK, children under the age of 16 can give consent for medical treatment once they are deemed able to fully appreciate what is involved (sometimes known as 'Gillick competence'). This is of growing importance for children whose parents have signed them up for genetic research and other studies that use their health data. When and how do children get a say in what happens to their health data?

Genomics England has been grappling with these complex questions for several years, recognizing that some of the participants in the 100,000 Genomes Project were recruited as children and are now reaching adulthood, while the youngest participants will not reach adulthood until the mid-2030s.

Thousands of UK families signed up for the 100,000 Genomes Project between 2015 and 2018. All of the families are affected by rare disease or cancer and have consented to share their whole genome sequences and associated UK National Health Service (NHS) health data with Genomics England, which curates and stores it in a trusted research environment (TRE). A TRE is akin to a vast reading library where approved researchers can come and investigate the data but are not allowed to take any of the data away. Research findings are reported in aggregate only, and no individual people can be identified. The ambition is for the Genomics England TRE (known as the 'National Genomic Research Library') to continue to grow in perpetuity, collecting longitudinal life-course data from NHS records about an increasing number of participants via the NHS Genomic Medicine Service, with the direct consent of participants.

Young people whose parents gave initial consent for their data to be included in the library will become adults between now and 2037 or thereabouts (no doubt moving to new homes and changing clinical teams along the way). It is imperative that participants are given the opportunity to choose whether they will continue to participate in this research, by re-consenting when the time is right, usually around age 16. If not, consent will lapse and no further data can be collated by the project team. This could have a substantial and increasing impact on the usefulness of the overall dataset over time.

One of the biggest challenges is to identify if or when young people will reach Gillick competence. Ultimately, doctors have the responsibility to decide whether a young person is competent to make their own healthcare decisions.

Many participants in genomics research have considerable learning difficulties and/or developmental delays, which may mean that their parents continue to be the primary decision-makers throughout their lives. Many other participants have very sound cognitive abilities, which will make it easy for them to decide what should happen with their data when they reach adulthood. Other participants will land somewhere in the middle, and without meeting them, it may be impossible to know if they are competent to consent. The subject of consent needs to be raised with the family - and the individual young person - in a tactful and sensitive way. Another challenge is how to obtain consent at scale, when tens of thousands of participants are affected.

There can be deep divisions of opinion between a young person with a health condition and their parents. Some may feel that their health condition adds to the richness of their character and they would not wish for it to be analyzed scientifically any further. Some may wish that they had more information about the condition; whether a cause or potential treatment can be identified. Others may feel they have seen no benefit from the research so far and do not see any value in keeping hopes alive any longer.
Researchers must tread carefully through such debates, recognizing the importance of presenting the re-consenting decision as neutrally as possible. No one should feel pressured into making a decision that they are in any way uncomfortable about.

One way to make this an easier task is to keep lines of communication open between the researchers, the participants, and the people who care for them, keeping the participants and caregivers up to date about the research that the health data are supporting. Without lifelong engagement, there is a considerable risk that when the young person reaches adulthood, and their consent is sought to continue, the response will be 'why?' rather than 'yes'. If the research project is still on the participant's radar, thanks to periodic communication from the researchers over the years, it is more likely that the participant will re-consent in the future.

Maintaining engagement in research studies can be as simple as sending a birthday card to participants each year, or sending a newsletter about the latest research that is using their health data. As well as engaging with participants, researchers should involve them through co-production, so that they can contribute to decisions about how their health data are being used. High-quality engagement and involvement of participants will increase the likelihood that more people will consent to continued use of their health data, which will benefit patients and researchers alike. $\square$

\section{Jillian Hastings Ward (D) \\ Participant Panel, Genomics England, London, UK.

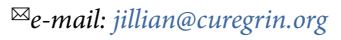

Published online: 6 January 2022 https://doi.org/10.1038/s41591-021-01561-w

Competing interests

J.H.W. is the independent chair of the Participant Panel at Genomics England; a lay member of the Science and Infrastructure Delivery Group at Health Data Research UK; a lay member of the Research Advisory Group at NHS Digital; a participant representative on the UK National Genomics Board; and a board member of the CureGRIN Foundation (https://curegrin.org/). 